\title{
A case study of gravity waves in noctilucent clouds
}

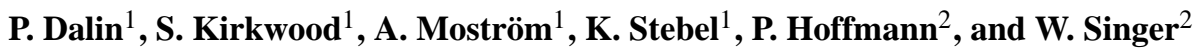 \\ ${ }^{1}$ Swedish Institute of Space Physics, Box 812, SE-981 28 Kiruna, Sweden \\ ${ }^{2}$ Leibniz Institute of Atmospheric Physics, Schloss-Straße 6, 18225 Kühlungsborn, Germany
}

Received: 29 September 2003 - Revised: 3 February 2004 - Accepted: 25 February 2004 - Published: 14 June 2004

\begin{abstract}
We present a case study of a noctilucent cloud (NLC) display appearing on 10-11 August 2000 over Northern Sweden. Clear wave structures were visible in the clouds and time-lapse photography was used to derive the parameters characterising the gravity waves which could account for the observed NLC modulation. Using two nearby atmospheric radars, the Esrange MST Radar data and Andoya MF radar, we have identified gravity waves propagating upward from the upper stratosphere to NLC altitudes. The wave parameters derived from the radar measurements support the suggestion that gravity waves are responsible for the observed complex wave dynamics in the NLC.
\end{abstract}

Key words. Atmospheric composition and structure (aerosols and particles; middle atmosphere-composition and chemistry) - Meteorology and atmospheric dynamics (middle atmosphere dynamics; waves and tides)

\section{Introduction}

Noctilucent clouds are a beautiful night-time optical phenomenon occurring in the mesopause region during the summer months at high latitudes. They can be seen normally from places within a latitude range from 48 to $70^{\circ}$ in the Northern Hemisphere (Gadsden and Schröder, 1989). The NLC appear in the twilight arch between about 22:00 and 04:00 local time (LT). The structure of NLC is similar to cirrostratus clouds but the former show more intensive and complex structures with signatures of waves of different scalesizes (partly due to differences in wave characteristics at the different heights and partly due to the larger area which is visible but also due to geometrical effects which result in better contrast). These waves create a wave-covered surface (sometimes on several levels) that moves as a whole, most often in the westward direction. In addition, some waves can propagate in different directions, disappearing and appearing

Correspondence to: P. Dalin

(pdalin@irf.se) again, and sometimes changing scale. A case study of such wave dynamics and the factors influencing the wave activity is the aim of this article.

The source of observed short wavelength structure (a few $\mathrm{km}$ ) in the NLC is thought to be Kelvin-Helmholtz waves caused by local wind shears (Hines and Reddy, 1967) while longer wavelengths $(20-70 \mathrm{~km})$ may be caused by internal gravity waves propagating from different directions (Hines, 1968; Witt, 1962). Short wavelength structures are generally quite unstable and their lifetime is of the order of ten minutes (Ludlam, 1957; Grishin, 1967). Long-period waves are rather stable and can persist for an hour or more. Tropospheric disturbances, which produce inertio-gravity waves propagating upward to NLC altitudes (Hines and Reddy, 1967), are considered likely to be responsible for long wavelength structures.

In this paper we firstly compare the observation of strong wave activity in the NLC with a gravity wave extracted from simultaneous wind measurements near NLC altitudes. The most interesting feature of this appearance is that the NLC were located close to an atmospheric radar in Sweden that continuously measures the vector wind velocity in the troposphere-lower stratosphere and in the vicinity of the mesopause. A second radar, located in Norway and quite close to the observed NLC, was also operating and provided vector wind velocities through most of the mesosphere. Simultaneous optical and radar observations allow us to estimate wave parameters in the NLC area and in its vicinity and to examine the interrelation between the observed wave modulation in the NLC and gravity waves propagating upward in the mesosphere.

\section{Optical observations of the NLC}

We consider the case of NLC appearance on 10-11 August 2000 above the northern part of Sweden (Fig. 1). Our observational site is located at the Lycksele ionospheric observatory in the north-eastern part of Sweden $\left(64^{\circ} 37^{\prime} \mathrm{N}\right.$; $18^{\circ} 44^{\prime} \mathrm{E}$ ). Observations of NLC were made by an automatic 

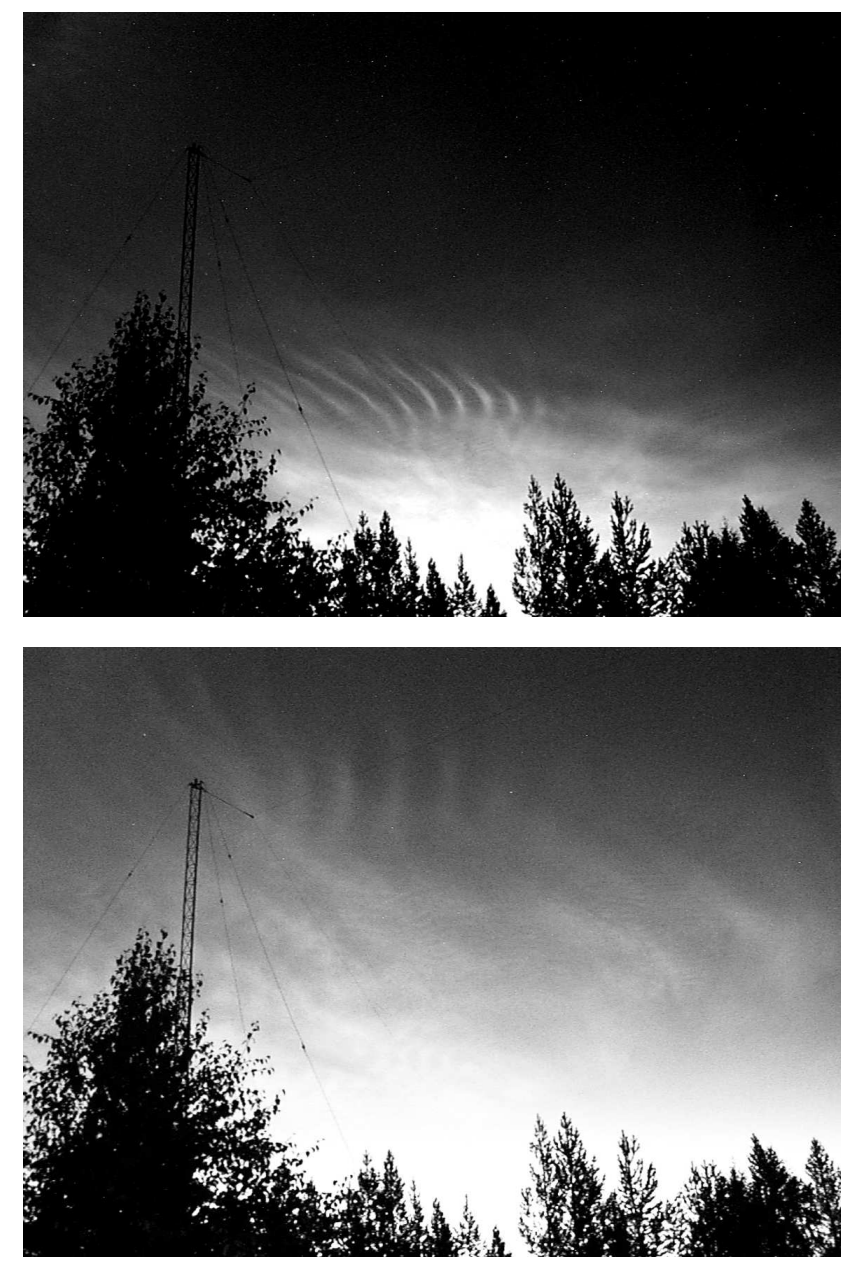

Fig. 1. NLC occurrence above the northern part of Sweden on 10 11 August 2000. Waves of various scales are presented.

CCD camera during the first two weeks of August. Earlier in the summer, the night sky was too bright for the NLC to be visible. The beginning and the end of the observations on 10-11 August were 23:00 and 03:00 LT. For this period the fixed camera took one frame per one minute, so whenever the NLC appeared, we have a perfect digital slide movie of the NLC movement. The CCD images have a dimension of $1280 \times 960$ pixels and an angular resolution of $0.038^{\circ}$ per pixel, equivalent to about a $0.20-0.33 \mathrm{~km}$ horizontal resolution for the NLC slant range of $300-500 \mathrm{~km}$.

To describe the NLC, we use the classification of morphological forms and the brightness estimation following the procedure elaborated by Grishin (1957). The weather conditions for this case were excellent, that is no tropospheric clouds were observed.

The NLC appeared at about 23:30 LT as white veils of brightness 1 with several short bands near perpendicular to the horizon between the azimuths $348^{\circ}-012^{\circ}$ at $11^{\circ}-17^{\circ}$ elevation angle. This area spread out and covered almost the whole twilight arch at 01:15 LT. The NLC moved from NE to SW (the borders and NLC displacements were defined by the angular characteristics of the NLC area using successive photographs). In addition, the bands became longer and their brightness increased. These bands represented clear ridges of a wave with a wavelength of about $9 \mathrm{~km}$ which propagated visually in the same direction as the whole NLC area. Meanwhile, other waves were created in the bottom part of the NLC area but the scales of these waves were different. There was a series of blurred bands, inclined to the horizon by 5$6^{\circ}$, with a relatively longer wavelength of about $20 \mathrm{~km}$ and those were crossed by small billows with wavelengths not more than $5 \mathrm{~km}$. During the NLC motion some bands and billows disappeared but at the same time new ones appeared. At 01:45 LT the NLC display reached its maximum area and the brightness reached the maximal value of 5 marks on the standard scale (estimated visually: 1 mark - very weak, 2 marks - weak, 3 marks - moderate, 4 marks - strong, 5 marks very strong brightness; Grishin, 1957). The NLC spread out to cover the whole twilight segment, and the NLC area represented a complex interference wave surface consisting of several types of waves of different scales from 5 to $40 \mathrm{~km}$. The upper boundary of the NLC was at $34^{\circ}$ above the horizon, and the left and right boundaries were located outside the field of view of the camera, extending at least from $340^{\circ}$ to $40^{\circ}$ of azimuth. The direction of the NLC movement as a whole coincided with the average direction of background wind at $83-86 \mathrm{~km}$ measured by the radar (see Fig. 2). After 01:55 LT the display became weaker and disappeared completely at about 02:30 LT on 11 August 2000.

It is interesting that no whirls (partial or complete rings of cloud with dark centres; Gadsden and Schröder, 1989) were observed for this display. This suggests that no turbulent structures were present and in this case any gravity waves present must have been quite stable, i.e. they did not reach saturation by an amplitude increase or by reaching a critical level at the NLC altitudes. This observational fact is very important and, as will be shown below, its interpretation is supported by the radar measurements.

\section{Radar observations}

Radar measurements of the signal-to-noise ratio (SNR) of the echo power, zonal, meridional and vertical components of velocity at different heights were made with both VHF and MF radars. The first radar, ESRAD, is located in the north of Sweden at the Esrange rocket range $\left(67^{\circ} 56^{\prime} \mathrm{N}, 21^{\circ} 04^{\prime} \mathrm{E}\right)$ and the second, the MF radar at Andenes, is placed in Northern Norway at the Andøya rocket range. $\left(69^{\circ} 17^{\prime} \mathrm{N}, 16^{\circ} 01^{\prime} \mathrm{E}\right)$. Detailed descriptions of these instruments and the measurement techniques can be found in Chilson et al. (1999) and in Singer et al. (1997), respectively. On this occasion, the ESRAD (VHF) radar measured wind parameters at heights of $2-12 \mathrm{~km}$ and $80-90 \mathrm{~km}$. Between 2 and $18 \mathrm{~km}$, the height and time resolution were $600 \mathrm{~m}$ and $12 \mathrm{~min}$, respectively. For the measurements in the mesosphere the height and temporal resolutions were set to $150 \mathrm{~m}$ and $2 \mathrm{~min}$. The Andenes MF radar measured over a height range of $50-98 \mathrm{~km}$ and its height/time resolution was $2 \mathrm{~km} / 2 \mathrm{~min}$. 


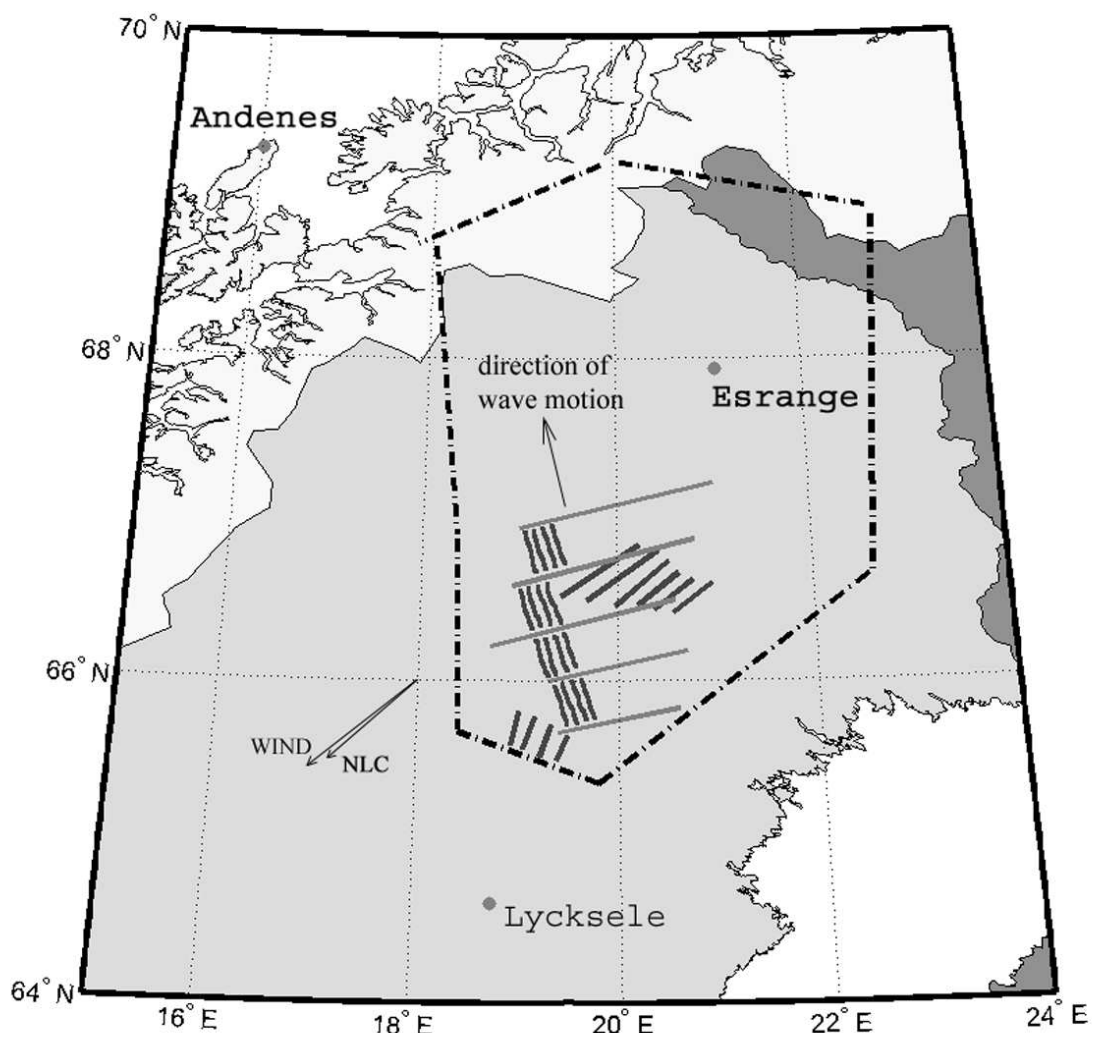

Fig. 2. Earth surface projection of the NLC area (dash) and observed waves of various scales (short and long lines).

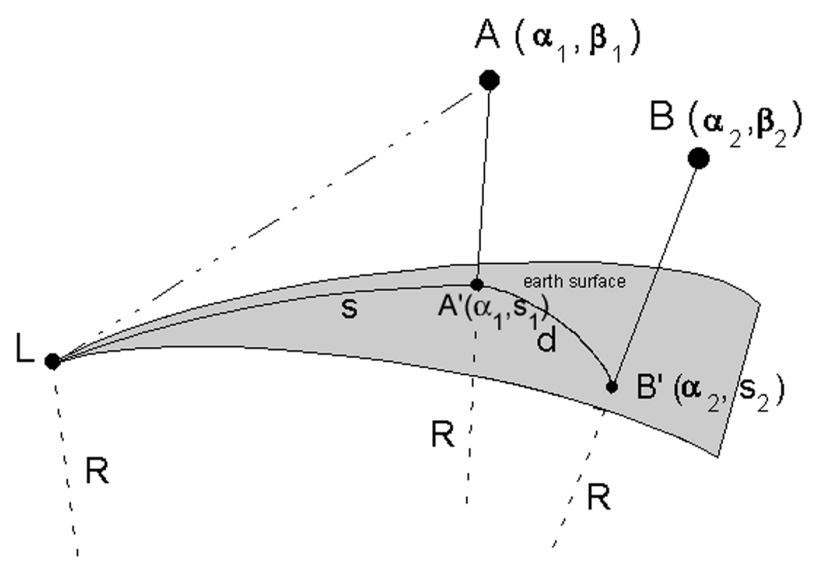

Fig. 3. Geometry of location of the observer (point L) and two points (A, B) in the NLC area.

\section{The estimation of wave parameters by photogram- metric technique}

In principle, in order to estimate the NLC wave scales and parameters from photographs, we would need observations from two points. In our case we have only single point observations from Lycksele. But nevertheless we can estimate wave characteristics because the altitude range of NLC is

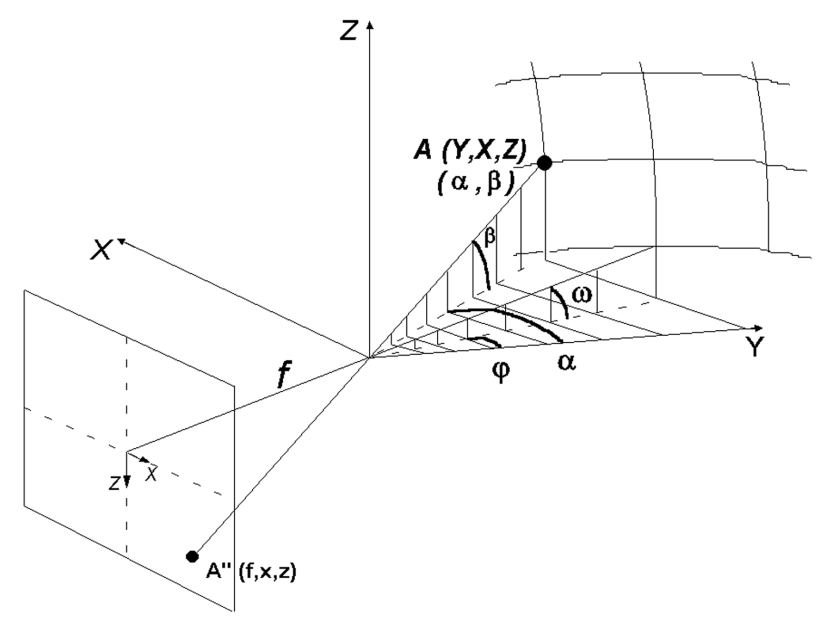

Fig. 4. Position of the NLC point $A$ and its projection $\left(A^{\prime \prime}\right)$ to the NLC image.

known. As has been shown in previous photogrammetric work (Witt, 1962; Burov, 1959) and as present lidar observations show (Nussbaumer et al., 1996) the average NLC altitude is quite stable at about $82-84 \mathrm{~km}$. Thus, it is adequate to use the median value of $82.9 \mathrm{~km}$ (Gadsden and Schröder, 1989) to estimate the wave characteristics in the NLC area. We don't insist on precise absolute values of those characteristics because the NLC altitude may vary as a whole and 
Table 1. Wave parameters in the NLC area estimated with photogrammetric technique.

\begin{tabular}{lr}
\hline Background wind velocity & $40-60 \mathrm{~m} / \mathrm{s}$ \\
Wavelength of small waves & $4-5 \mathrm{~km}$ \\
Wavelength of medium waves & $8-11 \mathrm{~km}$ \\
Wavelength of large waves & $33-47 \mathrm{~km}$ \\
Intrinsic horizontal phase velocity & $7-10 \mathrm{~m} / \mathrm{s}$ \\
Intrinsic period of large-scale waves & $55-112 \mathrm{~min}$ \\
Observed period of large-scale wave & $38-84 \mathrm{~min}$ \\
Intrinsic period of medium-scale waves & $13-18 \mathrm{~min}$ \\
Intrinsic period of small-scale waves & $6-8 \mathrm{~min}$ \\
\hline
\end{tabular}

also some details in the NLC area may change their height $( \pm 2 \mathrm{~km})$ due to the wave motion. Our aim is only to show that estimated parameters are of reasonable (typical) values for gravity waves and that these values are reflected in the wave activity in the surrounding area as seen by the radars.

In order to proceed we briefly describe the photogrammetric technique as applied to our case of single point observation. A full description for two points is given by Witt (1962), Burov (1959), Bronshten (1984).

Let us consider the camera located at the point $\mathrm{L}$ with known geographical coordinates $(\phi, \lambda)$. In order to measure the distance $d$ between two points A and B of the NLC (Fig. 3), located at the height $h$, we need to estimate the distances $s_{1}$ and $s_{2}$ from the point $\mathrm{L}$ to the points $\mathrm{A}^{\prime}$ and $\mathrm{B}^{\prime}$ projected to the Earth. Point A with the Cartesian coordinates $(x, y, z)$ has corresponding image coordinates $\mathrm{A}^{\prime \prime}(f, x, z)$ from the origin of the reference coordinate system, where $f$ is the focal length of the camera (Fig. 4).

Then the spherical coordinates (azimuth and elevation angle) of the point $\mathrm{A}^{\prime \prime}(\alpha, \beta)$ are defined as:

$\tan (\alpha-\varphi)=\frac{x}{f \cos \omega-z \sin \omega}$

$\tan \beta=\frac{f \sin \omega+z \cos \omega}{f \cos \omega-z \sin \omega} \cos (\alpha-\varphi)$

where $\omega$ is the angle of inclination of the optical axis to the horizon (elevation angle) and $\varphi$ is the azimuth of the optical axis of the camera. The spherical coordinates $(\omega, \varphi)$ of the origin of the reference coordinate system can be found if we know the spherical coordinates of control points in the picture (by theodolite measurements) or by using the coordinates of known stars. In our case we used the latter. If the coordinates axes of the camera are also rotated by an angle $\gamma$ (not shown in Fig. 4) to the perpendicular to the horizon, then in this case, first of all, we need to determine coordinates $(f$, $x, z)$ through coordinates $\left(f^{\prime}, x^{\prime}, z^{\prime}\right)$ in the rotated coordinate system:

$$
\begin{aligned}
& f=f^{\prime} \\
& x=x^{\prime} \cos \gamma+z^{\prime} \sin \gamma \\
& z=-x^{\prime} \sin \gamma+z^{\prime} \cos \gamma
\end{aligned}
$$

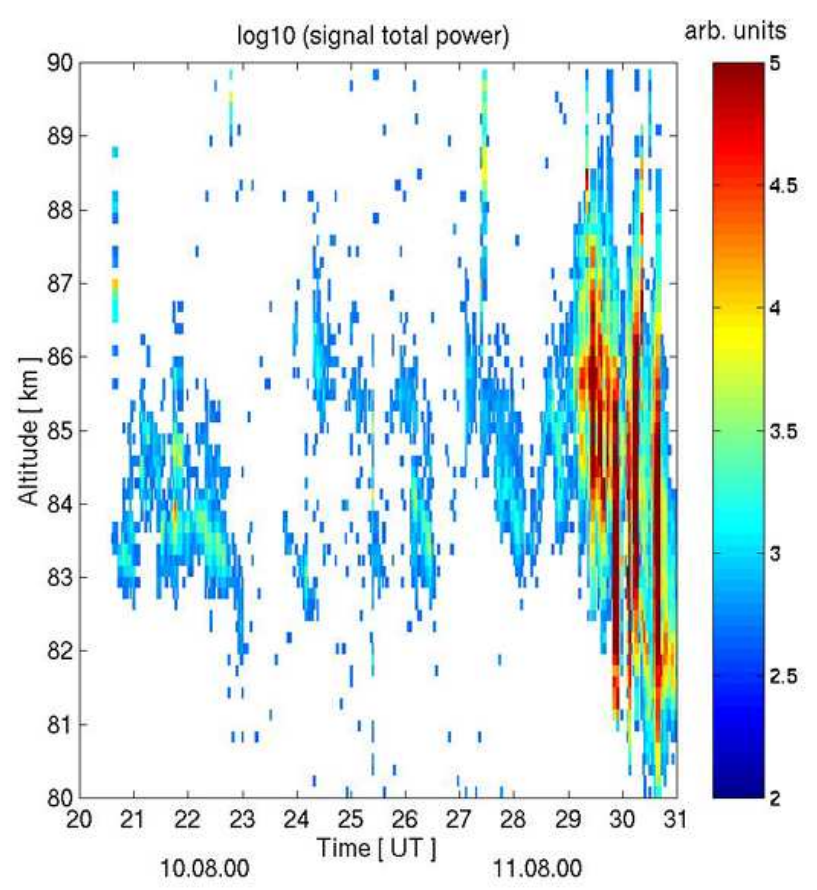

Fig. 5. Height-time distribution of the PMSE occurrence as the signal power at 2-min and 150-m resolution.

The distance $s_{1}$ from the observational point $\mathrm{L}$ to the projection of the NLC detail $\mathrm{A}^{\prime}$ can be found from the geometry of Fig. 3:

$$
s_{1}=R\left(\pi / 2-\beta^{\prime}-\arcsin \left(\frac{R \cos \beta^{\prime}}{R+h}\right)\right)
$$

where $\beta^{\prime}$ is the elevation angle of point A corrected for atmospheric refraction and $R=6389 \mathrm{~km}$ is the radius of meridian curvature for the latitudes of the observed NLC. Finally, the distance $d$ between two points $\left(s_{1}, \alpha_{1}\right)$ and $\left(s_{2}, \alpha_{2}\right)$ in the NLC area (Fig. 3) is given by:

$d=R \arccos \left(\cos s_{1} \cos s_{2}+\sin s_{1} \sin s_{2} \cos \left(\alpha_{1}-\alpha_{2}\right)\right)$

The velocity of the NLC detail is defined by its displacement during the time between two successive pictures. The accuracy of this estimation depends mainly on our assumption of the NLC altitude. If we put $\Delta h= \pm 2 \mathrm{~km}$, then the error in $\Delta d$ is $\pm 5 \mathrm{~km}$. Other errors in the spherical angle estimation are rather small and don't contribute more to the error than $\pm 0.5 \mathrm{~km}$. However, these are systematic errors that do not influence the velocity estimation.

Using successive photographs (one each minute), one can estimate both the movement of the NLC area as a whole and the displacement of wave crests relative to the background. To determine the direction and velocity of the NLC area we measured an average displacement of several areas located in different places of the NLC field apart from the obvious wave motion. Then, to find the intrinsic horizontal phase velocities and the direction of wave propagation the displacement 


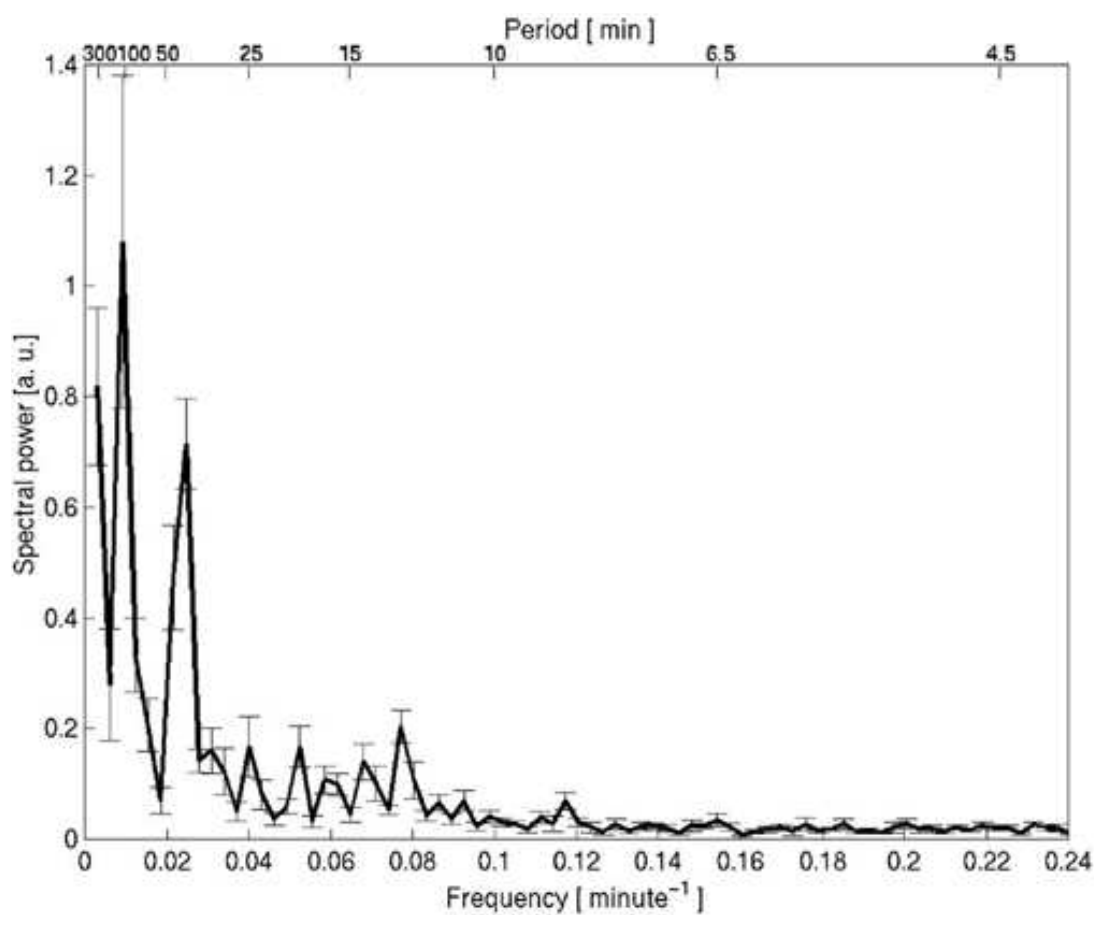

Fig. 6. Power spectrum of the PMSE in the range of $83-85 \mathrm{~km}$ with 2 -min and $150-\mathrm{m}$ resolution. Error bars are \pm twice the standard deviation divided by the square root of number of averages.

of wave crests relative to the NLC area as a whole were measured. This is possible because one can estimate the movement of such ambient areas as the veils that are less involved in the wave motion, so that we can assume that veils move mainly due to the background wind velocity at the appropriate height. Estimated parameters of the observed NLC modulation are listed in Table 1. These parameters are typical values for gravity wave motion observed close to the mesopause (Hines, 1968; Reid and Vincent, 1987) and in the next sections we investigate the wave activity at the heights below and try to find a possible relationship.

\section{Observation of gravity waves by radar measurements}

Previous work using radar measurements to investigate gravity waves with quite long horizontal wavelengths (several hundred $\mathrm{km}$ ) and long periods (several hours) has been reported by Cho (1995) and Sato (1994). In those cases, 30min averaged data were used. In our case we are interested in gravity waves of a smaller scale and therefore high resolution ( $2 \mathrm{~min}$ ) data were used.

In order to estimate gravity wave parameters we applied the Stokes-parameter spectra method and standard hodograph analysis, assuming the presence of a monochromatic gravity wave. The question of the validity of this assumption has been raised in several works (Eckermann and Hocking, 1989; Eckermann and Vincent, 1989). The hodograph analysis defines the wave parameters correctly if a monochromatic wave with large amplitude is present. As will be shown be- low, in our case, a quite regular velocity hodograph is defined when the effect of background wind shear has been taken into account. We have applied a simple hodograph analysis to Esrange MST radar data (above which the NLC were located) because, unfortunately, there were quite a lot of gaps in the measurements of velocity components at that period and therefore there was no possibility to apply the Fourier transform domain to sort out a monochromatic wave. The data gaps are due to the nature of the atmospheric structures causing the radar echoes, leading to only sporadic echoes during the month of August. Thus, we cannot definitively test for the presence of a monochromatic wave in the Esrange data. The same approach and procedure were examined by Rapp et al. (2002). They used falling sphere measurements (only one profile of zonal and meridional components) to extract a gravity wave. On the other hand, fortunately, the echoing structures are more continuous at MF wavelengths, and high resolution Andenes MF radar data are available. In this case we could apply the Stokes-parameter spectral method to estimate gravity wave characteristics.

Before estimating the wave characteristics from the radar winds, however, it is worthwhile to note that wave signatures are observed in the radar-echo intensity structures seen by ESRAD. These polar mesospheric summer echoes (PMSE) are thought to be caused by charged aerosols, including much smaller scale aerosols than those seen as NLC (Cho and Röttger, 1997; Rapp and Lübken, 2003). The PMSE appear in the same season and at the same altitudes as the NLC and they are a closely-related phenomenon (Nussbaumer et al., 1996; von Zahn and Bremer, 1999). Large-scale planetary 

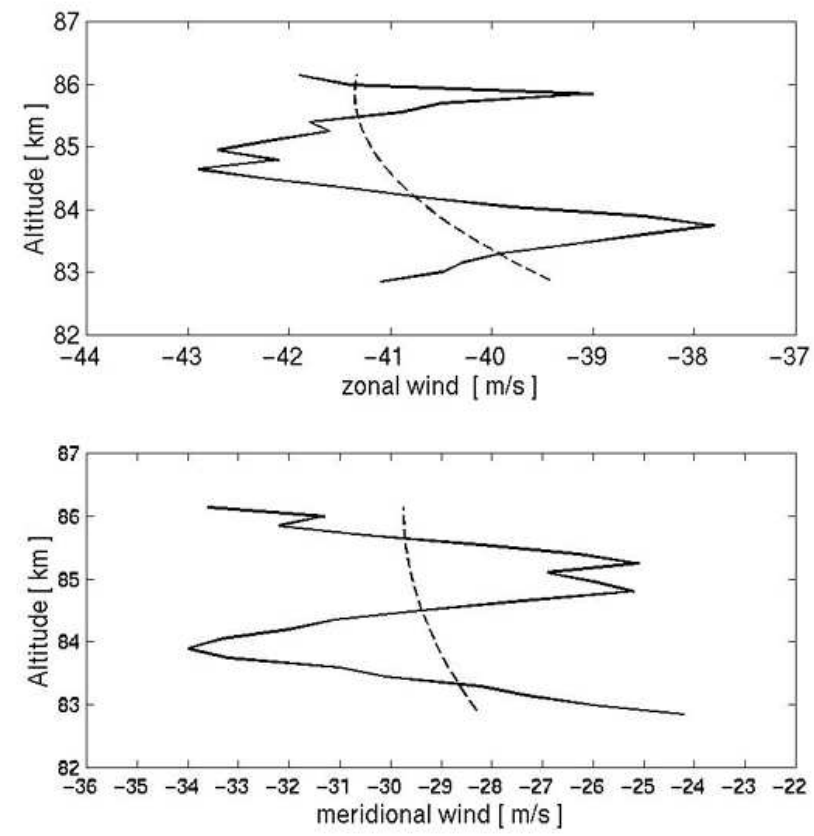

Fig. 7. Profiles of zonal (u) and meridional (v) component of wind velocity measured by ESRAD with $150-\mathrm{m}$ resolution at 22:35 UT on 10 August 2000. The background wind is marked by a dashed line.

wave modulation (4-6 days) has been found in PMSE (Kirkwood and Rechou, 1998). On the other hand, high resolution measurements $(2 \mathrm{~min})$ reveal substantially smaller periodicities. Figure 5 shows the PMSE occurrence as signal total power for our case study at heights $80-90 \mathrm{~km}$. Data from two main time intervals (between 20:30-23:00 UT and 00:0005:00 UT) have been examined by interpolating over short gaps, and combining into one data set. Several power spectra were taken with respect to time at different heights from 83 to $85 \mathrm{~km}$. The height-averaged spectrum is shown in Fig. 6 . Error bars are \pm twice the standard deviation divided by the square root of the number of averages. Two clear peaks are seen, corresponding to frequencies relative to the ground at periods of about $108 \mathrm{~min}$ and $41 \mathrm{~min}$. The first value is slightly out of the observed range periods of gravity waves estimated with the photogrammetric technique (see Table 1) but the second peak belongs to that interval. We suggest that this is a reflection of the propagation of gravity waves with appropriate observed periods. The period of $41 \mathrm{~min}$ is also close to that found in a radar/lidar/NLC analysis by Stebel et al. (2000). Thus, it has to be regarded as further evidence of the presence of gravity waves in the vicinity of the NLC for this case.

5.1 Estimation of gravity wave parameters with ESRAD measurements

The profiles of two components of wind velocity measured by ESRAD radar are shown in Fig. 7. We can see clear wave disturbances for zonal $\left(u^{\prime}\right)$, meridional $\left(v^{\prime}\right)$ components rela-

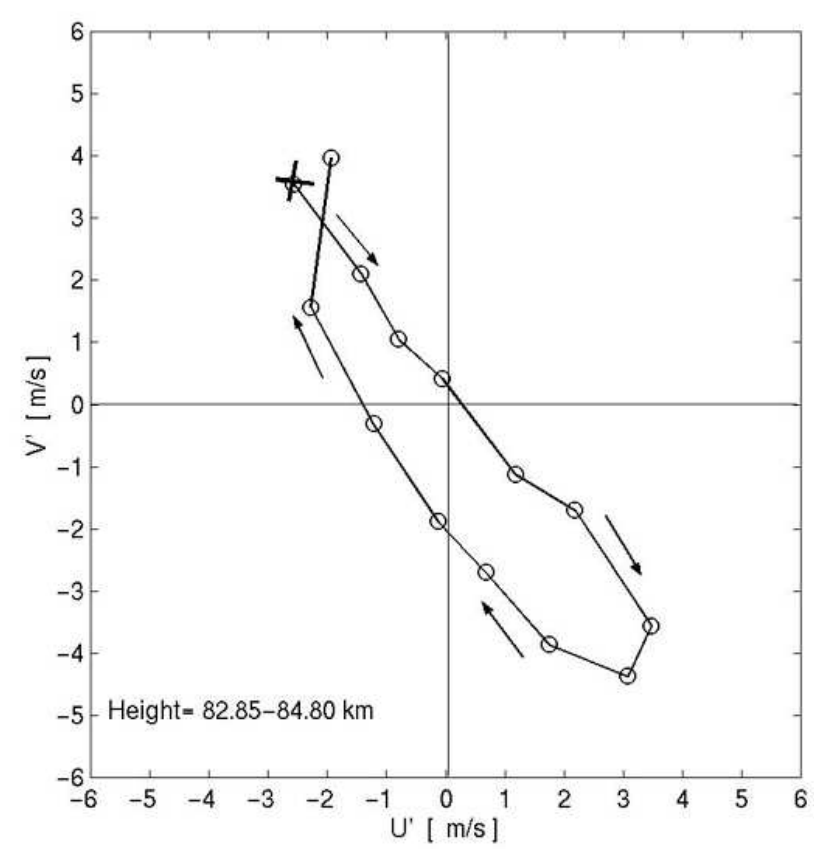

Fig. 8. Hodograph analysis of horizontal wind velocity disturbances with $150-$-m resolution at 22:35 UT on 10 August 2000.

tive to the background winds ( $\bar{u}, \bar{v}$ marked by dash line). The background wind components have been calculated by fitting the measurements using a second-order polynomial. The corresponding hodograph of the tip of the horizontal wind vector with respect to height (from 82.85 to $84.80 \mathrm{~km}$ ) is presented in Fig. 8. This hodograph has the form of an ellipse with high eccentricity. The horizontal perturbation wind vector has a clockwise rotation with increasing height which means that the group velocity of the gravity wave propagates upward (phase velocity is downward) in the Northern Hemisphere (Cot and Barat, 1986). With the hodograph one can estimate wave characteristics using the following equations (Hines, 1960; Cho, 1995).

The polarization relation is given by

$R=\frac{f}{\hat{\omega}}+\frac{1}{N} \frac{\partial \bar{v}}{\partial z}$

where $R$ is the magnitude of the ratio of the minor to major axis of polarization ellipse, $f$ the Coriolis parameter, $\hat{\omega}$ the intrinsic frequency of gravity wave, $N$ is the angular BruntVäisälä frequency, $\bar{v}$ the mean horizontal wind component perpendicular to the wave propagation and the term $\partial \bar{v} / \partial z$ represents the effect of vertical shear in the background wind field.

The dispersion relation is given by

$\hat{\omega}^{2}=f^{2}+\frac{N^{2} k^{2}}{m^{2}}-\frac{2 f k}{m} \frac{\partial \bar{v}}{\partial z}$

where $\mathrm{k}$ is the horizontal wave vector and $\mathrm{m}$ is the vertical wave number vector. This relation is valid under the condition $k^{2} \ll m^{2}$. 
Table 2. The characteristics of gravity wave estimated by the hodograph analysis (Esrange radar data).

\begin{tabular}{ll}
\hline $\mathrm{N}$ & $1.35 \cdot 10^{-4} \mathrm{rad} \mathrm{s}^{-1}$ \\
$\mathrm{R}$ & 0.20 \\
$d v / d z$ & $1.2 \cdot 10^{-3} \mathrm{~m} / \mathrm{s} / \mathrm{m}$ \\
$\Theta$ & $129^{\circ}$ northwards of east \\
$2 \pi / \hat{\omega}$ & $109 \mathrm{~min}$ \\
$2 \pi / \omega_{0}$ & $75 \mathrm{~min}$ \\
$2 \pi / \mathrm{m}$ & $-1.95 \mathrm{~km}$ \\
$2 \pi / \mathrm{k}$ & $41.5 \mathrm{~km}$ \\
$v_{p h}$ & $6.3 \mathrm{~m} / \mathrm{s}$ \\
$v_{p z}$ & $-0.30 \mathrm{~m} / \mathrm{s}$ \\
$v_{g h}$ & $6.2 \mathrm{~m} / \mathrm{s}$ \\
$v_{g z}$ & $0.29 \mathrm{~m} / \mathrm{s}$ \\
\hline
\end{tabular}

The Doppler relation is:

$\omega_{0}=\hat{\omega}+\bar{u} k$

where $\omega_{0}$ is the gravity wave frequency observed by the radar, $\bar{u}$ the mean horizontal wind component parallel to the wave propagation.

Next, the horizontal and vertical components of the phase velocity are defined as:

$v_{p h}=\hat{\omega} / k$

$v_{p z}=\hat{\omega} / m$

and the horizontal and vertical components of the group velocity are defined as:

$v_{g h}=\partial \hat{\omega} / \partial k$

$v_{g z}=\partial \hat{\omega} / \partial m$.

Solving this equation system one can find the wave characteristics.

The value of the Coriolis parameter for ESRAD's location is $1.35 \times 10^{-4} \mathrm{~s}^{-1}$. $N$ has been taken as $0.020 \mathrm{~s}^{-1}$ for the mesopause following the general consideration by Hines (1968), Witt (1962) and temperature measurements by Lübken (1999).

The polarisation ratio of the ellipse under consideration is equal to 0.20 . Thus, it is very important to take wind shear into account as even small values for this parameter can affect the resulting value for the intrinsic frequency of the gravity wave. Although the absolute value of the wind shear in our case is relatively small $\left(1.2 \times 10^{-3} \mathrm{~m} / \mathrm{s} / \mathrm{m}\right)$, the term $\frac{1}{N} \frac{\partial \bar{v}}{\partial z}$ amounts to $26 \%$ of the observed ratio $R$. This large effect is because the background wind is near perpendicular to the orientation of the major axis of the ellipse and therefore to the wave propagation. Note that the major axes orientation is nearly the same direction as the orientation of the wave vector estimated from the NLC photographs. We chose the sign $k$ in which the value of observed frequency $\omega_{0}$ is closest to $\omega_{0}$ estimated directly from the photogrammetric technique and from the PMSE spectrum. In this way $k$ is positive and, in turn, this means that the wave moves to the north-west, as
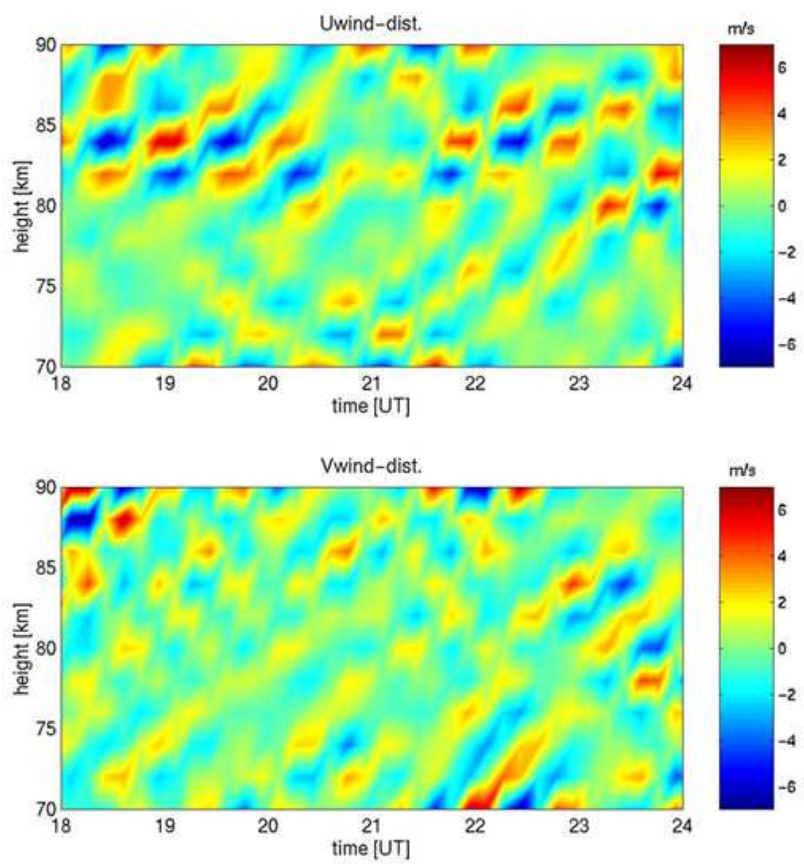

Fig. 9. Height-time distributions of zonal and meridional wind disturbances by Andenes MF radar measurements on 10 August 2000. Band-pass digital filtering both in the vertical direction and in time was applied to the original wind components.

was the case for the wave found by analysing the NLC photographs. The gravity wave parameters are listed in Table 2.

We can note that these estimated parameters are in reasonable agreement with ones obtained by the photogrammetric technique. In reality the horizontal wavelength and the intrinsic period $(2 \pi / \hat{\omega})$ are just in the range of the corresponding values of Table 1, and the intrinsic horizontal phase velocity (by the radar) is nearly the same as the values found with the NLC photographs.

The radar data analysis supports the view that the gravity wave for this case does not reach saturation by amplitude. The amplitude of this wave is $5.3 \mathrm{~m} / \mathrm{s}$, less than the intrinsic phase velocity of $6.3 \mathrm{~m} / \mathrm{s}$. Thus, we can see that the gravity wave, once initiated, could propagate upward at least through the region covered by the ESRAD radar measurements. It does not appear to have been filtered by the background wind in this region because the latter is near orthogonal to the gravity wave propagation.

\section{Gravity wave parameter estimation with Andenes radar measurements}

The Andenes MF radar is located in Northern Norway (Andøya) at $69^{\circ} 17^{\prime} \mathrm{N} ; 16^{\circ} 01^{\prime} \mathrm{E}$. For the case considered here, reliable horizontal wind velocity measurements are available for 70-90 km with height-time resolutions of $2 \mathrm{~km}$ and $2 \mathrm{~min}$. We used these high time resolution data to test the possibility of short periodicities in the original data. 
Table 3. Gravity wave parameters estimated from Andenes MF radar data.

\begin{tabular}{ll}
\hline$d v / d z$ & $1.3 \cdot 10^{-3} \mathrm{~m} / \mathrm{s} / \mathrm{m}$ \\
$N$ & $1.37 \cdot 10^{-4} \mathrm{rad} \mathrm{s}^{-1}$ \\
$R$ & 0.31 \\
$\Theta$ & $33^{\circ}$ (northwards of east) \\
$2 \pi / m$ & $-5.5 \mathrm{~km}$ \\
$2 \pi / k$ & $169 \mathrm{~km}$ \\
$2 \pi / \hat{\omega}$ & $3.0 \mathrm{~h}$ \\
$2 \pi / \omega_{0}$ & $1.2 \mathrm{~h}$ \\
$v_{p h}$ & $15.6 \mathrm{~m} / \mathrm{s}$ \\
$v_{p z}$ & $-0.51 \mathrm{~m} / \mathrm{s}$ \\
$v_{g h}$ & $14.5 \mathrm{~m} / \mathrm{s}$ \\
$v_{g z}$ & $0.47 \mathrm{~m} / \mathrm{s}$ \\
\hline
\end{tabular}

Due to the height resolution of $2 \mathrm{~km}$, with the Andenes MF radar we can resolve only vertical wavelength larger than $4 \mathrm{~km}$, corresponding to horizontal wavelength larger than $100 \mathrm{~km}$ for medium-frequency waves $(f \ll \hat{\omega} \ll N)$, whose period may be more precisely estimated with the dispersion relation. Thus, a priori, there is little chance to find the same high-frequency and small-scale wave modulation simultaneously in both Andenes and ESRAD data. But nevertheless we regarded Andenes measurements as describing general wave conditions in the mesosphere for our case of NLC occurrence.

To reduce the statistical/geophysical variability, wind velocity variation data of 2-min resolution were averaged over $10 \mathrm{~min}$ at each height. Then, Fourier transforms of zonal and meridional components were taken with respect to altitude and time and next, a filtering procedure was applied to reveal possible variations with periods of $30 \mathrm{~min}-2 \mathrm{~h}$ and with vertical wavelengths of 4-11 km.

The height-time distributions of zonal and meridional wind disturbances on 10 August 2000 are shown in Fig. 9. A band-pass digital filter both in the vertical direction and in time was applied to the original wind components to obtain this plot. Clear wave-like modulations with a period a little bit more than $1 \mathrm{~h}$ and with a 5-7 km vertical wavelength are observed in both zonal and meridional components between 21:30 and 23:30 UT in the height range of 70-90 km. To quantify these periodicities we have applied the Stokesparameter spectra technique following the procedure by Eckermann (1996), Eckermann and Vincent (1989), Vincent and Fritts (1987). This technique assumes that given vertical profiles of horizontal velocity perturbations $u^{\prime}(z)$ and $v^{\prime}(z)$ may contain several harmonics. Then, Fourier transforming them over their full height ranges yields complex components:

$\boldsymbol{U}(m)=U_{R}(m)+i U_{I}(m)$

$\boldsymbol{V}(m)=V_{R}(m)+i V_{I}(m)$ where $m$ is the vertical wave number. Then, power spectral densities for standard four Stokes parameters $(I, D, P, Q)$ are defined as:

$$
\begin{aligned}
& \tilde{I}(m)=A\left(\overline{U_{R}^{2}(m)}+\overline{U_{I}^{2}(m)}+\overline{V_{R}^{2}(m)}+\overline{V_{I}^{2}(m)}\right) \\
& \tilde{D}(m)=A\left(\overline{U_{R}^{2}(m)}+\overline{U_{I}^{2}(m)}-\overline{V_{R}^{2}(m)}-\overline{V_{I}^{2}(m)}\right) \\
& \tilde{P}(m)=2 A\left(\overline{U_{R}(m) V_{R}(m)}+\overline{U_{I}(m) V_{I}(m)}\right) \\
& \tilde{Q}(m)=2 A\left(\overline{U_{R}(m) V_{I}(m)}-\overline{U_{I}(m) V_{R}(m)}\right)
\end{aligned}
$$

where $A$ is a scaling constant, and overbars denote averages over a number of independent spectra, to remove the effects of incoherent motions. The degree of polarization of the wave $\tilde{d}(m)$ is defined as follows:

$\tilde{d}^{2}(m)=\frac{\tilde{D}^{2}(m)+\tilde{P}^{2}(m)+\tilde{Q}^{2}(m)}{\tilde{I}^{2}(m)}$

$\tilde{d}(m)$ is also rotationally invariant to coordinate axes of the ellipse, the value of $\tilde{d}(m)$ changes in the range of $0 \div 1$. Parameter $\tilde{d}(m)$ is an analog to the cross-spectrum value $\tilde{S}(m)$ but the former does not depend on the rotation of semimajor axis of the wave ellipse and therefore it represents better the wave-variance content (for more details, see Eckermann, 1996). Then, the phase of ellipticity $\tilde{\Phi}(m)$, the orientation of semimajor axes of the ellipse $\tilde{\Theta}(m)$ and axial ratio $\tilde{R}(m)$ are defined by the following relations (Eckermann and Vincent, 1989):

$$
\begin{aligned}
& \tilde{\Phi}(m)=\arctan \frac{\tilde{Q}(m)}{\tilde{P}(m)} \\
& \tilde{\Theta}(m)=\frac{1}{2} \arctan \frac{\tilde{P}(m)}{\tilde{D}(m)} \\
& \tilde{R}(m)=\tan \left(\frac{1}{2} \arcsin \left(\frac{\tilde{Q}(m)}{\tilde{d}(m) \cdot \tilde{I}(m)}\right)\right)
\end{aligned}
$$

These parameters $(\tilde{d}(m), \tilde{\Phi}(m), \tilde{\Theta}(m))$ estimated for our case are shown in Fig. 10. Error bars are \pm twice the standard deviation divided by the square root of the number of averages. The clear maximum for function $\tilde{d}(m)$ takes place for the vertical wave number $m=1.14 \mathrm{~km}^{-1}$ or vertical wavelength $\lambda_{x}=5.5 \mathrm{~km}$. The sign of phase is positive, which means that the gravity wave propagates upward. The value of phase belongs to an elliptical polarization and the orientation of the semimajor axes is in the I-III quadrant. Note that the standard deviations for all estimated parameters are small for that particular wave number.

Using Eqs. (6-10) we have estimated gravity wave characteristics and they are listed in Table 3. Note we have included the horizontal wind shear effect in the calculations.

This is a medium-scale wave which propagated upward and with NE-SW azimuth, that is in the direction of an average wind. The direction of motion was defined so that the 

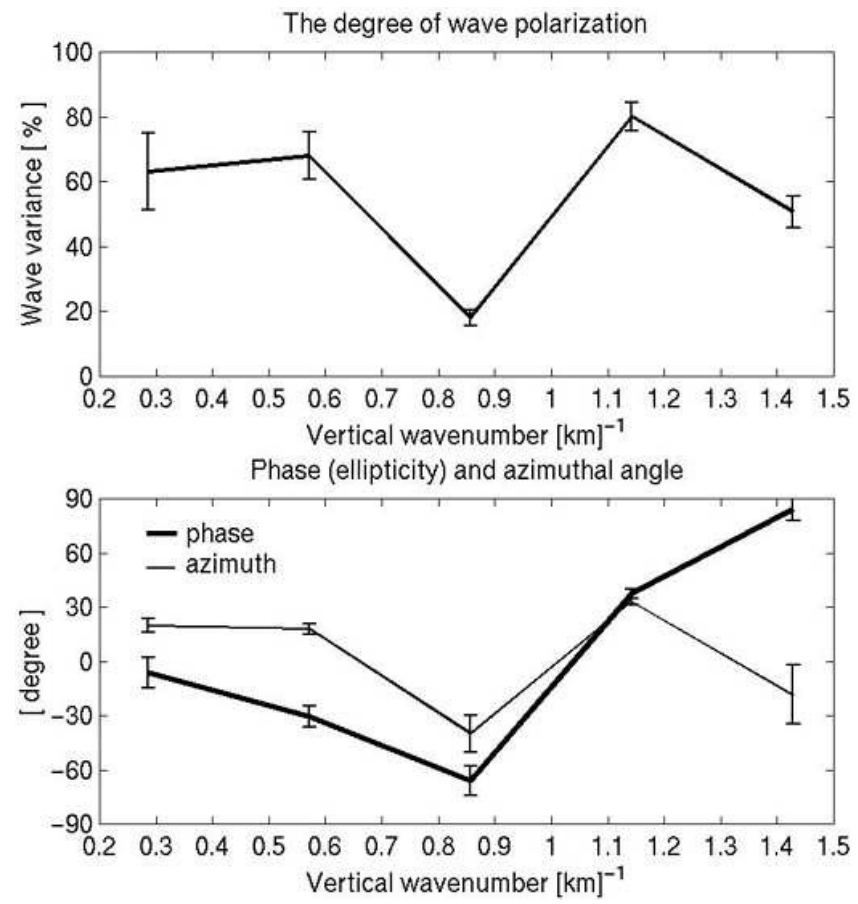

Fig. 10. Upper panel: The degree of wave polarization $\tilde{d}(m)$. Lower panel: ellipticity $(\tilde{\Phi}(m)$ thick line $)$ and azimuth angle $(\tilde{\Theta}(m)$ thin line) of the gravity wave obtained by Stokes-parameter spectra technique for wind disturbances (Andenes MF radar) between 21:30 23:30 UT on 10 August 2000 and for the height range of 70-90 km.

observed period was closest to that directly estimated from the filtering analysis.

To support the general consideration, the hodograph analysis was applied to the $u^{\prime}$ and $v^{\prime}$ components. The horizontal velocity vector rotation with height for 22:00 UT (10 August 2000) is shown in Fig. 11. This vector has a clear clockwise rotation through all of the height range from 70 to $90 \mathrm{~km}$ (three cycles with 3-4 points), representing a vertical wavelength in the range of $6-8 \mathrm{~km}$. It seems that this wave did not reach saturation because the amplitude of the horizontal wind component of a gravity wave (3-4 m/s) was less than the magnitude of the intrinsic horizontal phase velocity $(15.6 \mathrm{~m} / \mathrm{s})$ at any altitude. We note that the waves extracted from Andenes MF radar data are not the same waves as found from the Esrange radar data. Andenes data do not allow us to sort out small vertical wavelengths less than $4 \mathrm{~km}$ but we show that general conditions were favorable for gravity waves propagating upward through the mesosphere region.

\section{Conclusions}

We have considered one case of observation of gravity waves in NLC observed above Sweden. Time-lapse camera observations together supplement radar wind measurements and show the clear presence of medium-scale gravity waves. Such NLC observations can therefore be very useful for the

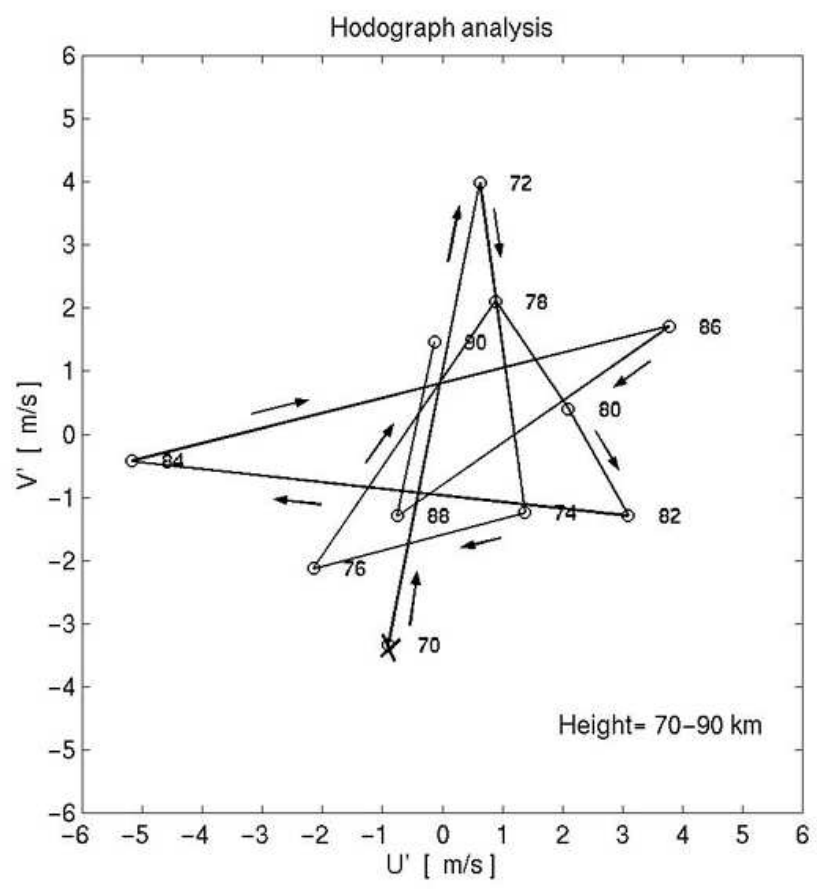

Fig. 11. Hodograph analysis of Andenes wind disturbances between $70-90 \mathrm{~km}$ at 22:00 UT on 10 August 2000.

analysis of gravity-wave dynamics even when the NLC are observed from only one point.

As both radar and optical observations are conducted regularly (the latter each year during the NLC season), one can hope to register several occasions of the presence of gravity waves in the NLC area and therefore to obtain statistical information on typical wave characteristics in the upper mesosphere-mesopause system above a given region of the Earth's surface. Based on the knowledge of typical wave parameters and wave orientations we can attempt to define a possible source for these waves (such as likely an orographic source represented by the Scandinavian ridge). Relating sources to conditions in the troposphere will contribute to a better understanding of the mechanisms of gravity wave forcing in the summer mesosphere. A comprehensive characterization of the sources of gravity waves, with paths traced to the mesopause altitudes, would require multipoint ground-based radar measurements accompanied by simultaneous sounding rocket experiments, as well as standard global meteorological assimilations. However, some progress can likely be made on the basis of the radar used here, together with meteorological assimilations when local sources dominate.

In the case considered both optical and Esrange radar observations revealed a gravity wave with approximately the same characteristics propagating from the south-east to the north-west direction. This direction coincides with that found by Vincent and Fritts (1987) for medium-period waves in the summer season. The following results regarding wave characteristics are obtained: 
1. Optical observations show that a gravity wave with an intrinsic period of about $1.5 \mathrm{~h}$ and with a horizontal wavelength of about $40 \mathrm{~km}$ propagated in the vicinity of the mesopause from south-east to north-west. Its phase velocity relative to the background was $7-10 \mathrm{~m} / \mathrm{s}$.

2. Simultaneous Esrange radar measurements revealed a gravity wave with an intrinsic period of $1.8 \mathrm{~h}$, with a horizontal wavelength of about $40 \mathrm{~km}$ and with vertical wavelength of $2 \mathrm{~km}$. This wave propagated upward with a vertical phase velocity of $0.3 \mathrm{~m} / \mathrm{s}$. The horizontal phase velocity was $6.3 \mathrm{~m} / \mathrm{s}$ and the wave did not reach the critical level.

3. Simultaneous Andenes MF radar measurements show another type of gravity wave, with a longer period of about $3 \mathrm{~h}$, with a horizontal wavelength of about $170 \mathrm{~km}$ and a vertical wavelength of $6 \mathrm{~km}$. This wave propagated upward through the mesosphere and did not reach saturation.

Acknowledgements. ESRAD is jointly operated by the Swedish Institute of Space Physics and Swedish Space Corporation (ESRANGE). The main funding for this project has been from the Swedish Research Council. We are grateful to D. Fritts for very useful comments and discussions during the manuscript preparation.

Topical Editor U.-P. Hoppe thanks a referee for his help in evaluating this paper.

\section{References}

Bronshten, V. A.: Noctilucent clouds and their observations, (in Russian), Nauka, Moscow, 128, 1984.

Burov, M. I.: Methode photogrammetrique pour la determination de l'altitude des nuages argentes, (in Russian), Trudy soveschaniya po serebristym oblakam, Tartu, 92-111, 1959.

Chilson, P., Kirkwood, S., and Nilsson, A.: The Esrange MST radar: a brief introduction and procedure for range validation using balloons, Radio Sci., 34, 427-436, 1999.

Cho, J. Y. N.: Inertio-gravity wave parameter estimation from crossspectral analysis, J. Geophys. Res., 100, D9, 18727-18737, 1995.

Cho, J. Y. N. and Röttger, J.: An updated review of polar mesosphere summer echoes: observations, theory, and their relationship to noctilucent clouds and subvisible aerosols, J. Geophys. Res., 102, 2001-2020, 1997.

Cot, C. and Barat, J.: Wave-turbulence interaction in the stratosphere: a case study, J. Geophys. Res., 91, D2, 2749-2756, 1986.

Eckermann, S. D. and Hocking, W. K.: Effect of superposition on measurements of atmospheric gravity waves: a cautionary note and some reinterpretations, J. Geophys. Res., 94, 6333-6339, 1989.

Eckermann, S. D. and Vincent, R. A.: Falling sphere observations of anisotropic gravity wave motions in the upper stratosphere over Australia, Pure Appl. Geophys., 130, 509-532, 1989.
Eckermann, S. D.: Hodographic analysis of gravity waves: relationships among Stokes parameters, rotary spectra and cross-spectral methods, J. Geophys. Res., 101, 19 169-19 174, 1996.

Gadsden, M. and Schröder, W.: Noctilucent Clouds, SpringerVerlag, New York, 165 pp., 1989.

Grishin, N. I.: Instructions for noctilucent cloud observations, (in Russian), Izdatelstvo Akad. Nauk SSSR, Moscow, 23, 1957.

Grishin, N. I.: Morphological investigations and the nature of noctilucent clouds, (in Russian), in "Observations of noctilucent clouds", Izdatelstvo Nauka, 5-34, 1967.

Hines, C. O.: Internal atmospheric gravity waves at ionospheric heights, Can. J. Phys., 38, 1441-1481, 1960.

Hines, C. O. and Reddy, C. A.: On the propagation of atmospheric gravity waves through regions of wind shear, J. Geophys. Res., 72, 3, 1015-1034, 1967.

Hines, C. O.: A possible source of waves in the noctilucent clouds, J. Atmos. Sci., 25, 937-942, 1968.

Kirkwood, S. and Rechou, A.: Planetary-wave modulation of PMSE, Geophys. Res. Lett., 25, 24, 4509-4512, 1998.

Ludlam, F. H.: Noctilucent clouds, Tellus IX, 3, 341-364, 1957.

Lübken, F.-J.: Thermal structure of the Arctic summer mesosphere, J. Geophys. Res., 104, D8, 9135-9149, 1999.

Nussbaumer, V., Fricke, K.-H., Langer, M., Singer, W., and von Zahn, U.: First simultaneous and common volume observations of noctilucent clouds and polar mesosphere summer echoes by lidar and radar, J. Geophys. Res., 101, 19 161-19 167, 1996.

Rapp, M. and Lübken, F.-J.: On the nature of PMSE: electron diffusion in the vicinity of charged particles revisited, J. Geophys. Res., 108, D8, 8437, doi:10.1029/2002JD002857, 2003.

Rapp, M., Lübken, F.-J., Müllemann, A., Thomas, G. E., and Jensen, E. J.: Small scale temperature variations in the vicinity of NLC: experimental and model results, J. Geophys. Res., 107, doi:10.1029/2001JD001241, 2002.

Reid, I. A. and Vincent, R. A.: Measurements of the horizontal scales and phase velocities of short period mesospheric gravity waves at Adelaide, Australia, J. Atmos. Terr. Phys., 49, 10, 10331048, 1987.

Sato, K.: A statistical study of the structure, saturation and sources of inertio-gravity waves in the lower stratosphere observed with the MU radar, J. Atmos. Terr. Phys., 56, 6, 755-774, 1994.

Singer, W., Keuer, D., and Eriksen, W.: The ALOMAR MF radar: technical design and first results, in Proceedings of the 13th ESA Symposium on European Rocket and Balloon Programmes and Related Research, Oeland, Sweden (ESA SP-397), 101-103, 1997.

Stebel, K., Barabash, V., Kirkwood, S., Siebert, J., and Fricke, K.H.: Polar mesosphere summer echoes and noctilucent clouds: simultaneous and common-volume observations by radar, lidar and CCD camera, Geophys. Res. Lett., 27, 5, 661-664, 2000.

von Zahn, U. and Bremer, J.: Simultaneous and common-volume observations of noctilucent clouds and polar mesospheric summer echoes, Geophys. Res. Lett., 11, 1521-1524, 1999.

Vincent, R. A. and Fritts, D. C.: A climatology of gravity wave motions in the mesopause region at Adelaide, Australia, J. Atmos. Sci., 44, 4, 748-760, 1987.

Witt, G.: Height, structure and displacements of noctilucent clouds, Tellus XIV, 1, 1-18, 1962. 